\title{
BESOV-LIPSCHITZ AND MEAN BESOV-LIPSCHITZ SPACES OF HOLOMORPHIC FUNCTIONS ON THE UNIT BALL
}

\author{
MIROLJUB JEVTIĆ AND MIROSLAV PAVLOVIĆ
}

\begin{abstract}
We give several characterizations of holomorphic mean BesovLipschitz space on the unit ball in $\mathbb{C}^{N}$ and appropriate Besov-Lipschitz space and prove the equivalences between them. Equivalent norms on the mean Besov-Lipschitz space involve different types of $L^{p}$-moduli of continuity, while in characterizations of Besov-Lipschitz space we use not only the radial derivative but also the gradient and the tangential derivatives. The characterization in terms of the best approximation by polynomials is also given.
\end{abstract}

\section{INTRODUCTION AND MAIN RESULTS}

Let $\mathbb{B}_{N}$ denote the unit ball in $\mathbb{C}^{N}$ and let $\mathbb{S}_{N}=\partial \mathbb{B}_{N}$, where $N$ is a positive integer. For a point $z=\left(z_{1}, \ldots, z_{N}\right) \in \mathbb{C}^{N}$ we write $|z|=\left(\left|z_{1}\right|^{2}+\ldots+\left|z_{N}\right|^{2}\right)^{1 / 2}$. The normalized Lebesgue measures on $\mathbb{B}_{N}$ and $\mathbb{S}_{N}$ will be denoted by $d v$ and $d \sigma$, respectively. The $L^{p}$-mean over the sphere $|z|=r(0<r<1)$ of a Borel function $f$ on $\mathbb{B}_{N}$ is defined by

$$
M_{p}(r, f)=\left(\int_{\mathbb{S}_{N}}|f(r \zeta)|^{p} d \sigma(\zeta)\right)^{1 / p} \quad(1 \leq p<\infty)
$$

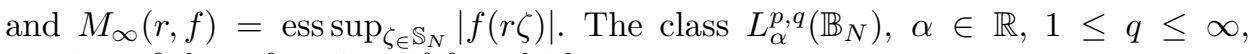
consists of those functions $f$ for which

$$
\|f\|_{p, q, \alpha}:=\left(2 N \int_{0}^{1} M_{p}^{q}(r, f)\left(1-r^{2}\right)^{q \alpha-1} r^{2 N-1} d r\right)^{1 / q}<\infty .
$$

In the case $q=\infty$ this should be interpreted as

$$
\|f\|_{p, \infty, \alpha}:=\underset{0 \leq r<1}{\operatorname{ess} \sup }\left(1-r^{2}\right)^{\alpha} M_{p}(r, f)<\infty .
$$

Let $H\left(\mathbb{B}_{N}\right)$ denote the space of all holomorphic functions in $\mathbb{B}_{N}$. For $1 \leq p \leq \infty$ the Hardy space $H^{p}\left(\mathbb{B}_{N}\right)=H^{p}$ consists of all functions $f \in H\left(\mathbb{B}_{N}\right)$ such that

$$
\|f\|_{p}:=\sup _{0<r<1} M_{p}(r, f)<\infty
$$

i.e. $H^{p}\left(\mathbb{B}_{N}\right)=L_{0}^{p, \infty}\left(\mathbb{B}_{N}\right) \cap H\left(\mathbb{B}_{N}\right)$. For information on Hardy spaces of several variables we refer to 20 .

We are interested in the mixed-norm Bergman space

$$
H^{p, q, \alpha}=H^{p, q, \alpha}\left(\mathbb{B}_{N}\right)=L_{\alpha}^{p, q} \cap H\left(\mathbb{B}_{N}\right)
$$

2000 Mathematics Subject Classification. 32A35, 32A36, 32A37.

Key words and phrases. Besov-Lipschitz spaces, mixed-norm Bergman spaces, radial derivatives.

The authors are supported by MN Serbia, Project ON144010. 
and some closely related spaces. Note that $H^{p, q, \alpha}=\{0\}$ for $\alpha \leq 0$, if $1 \leq q<\infty$, and for $\alpha<0$ if $q=\infty$. By using standard arguments one proves that $H^{p, q, \alpha}$ is complete. These spaces arise naturally in the study of Hardy spaces (see [7]). Note also that $(1.1)$, for $1 \leq p=q<\infty$, reduces to

$$
\|f\|_{p, p, \alpha}=\int_{\mathbb{B}_{N}}|f(z)|^{p}\left(1-|z|^{2}\right)^{p \alpha-1} d v(z) .
$$

Therefore, $H^{p, p, \alpha}$ is a weighted Bergman space.

Convention. From now on, unless specified otherwise, we will assume $1 \leq p, q \leq$ $\infty, \alpha>0$, and $\nu, N$ are positive integers.

1.1. Besov-Lipschitz spaces. In order to give one of several possible definitions of Besov-Lipschitz spaces we need some more notation. The radial derivative $\mathcal{R} f$, where $f \in H\left(\mathbb{B}_{N}\right)$, is defined as

$$
\mathcal{R} f(z)=\sum_{j=1}^{N} z_{j} \frac{\partial f}{\partial z_{j}}(z),(*)
$$

which can also be written as

$$
\mathcal{R} f(z)=\sum_{k=1}^{\infty} k f_{k}(z),
$$

where $f(z)=\sum_{k=0}^{\infty} f_{k}(z)$ is the homogeneous expansion of $f$. Using the homogeneous expansion we can define $\mathcal{R}^{s} f$ for any $s \in \mathbb{C}$ by

$$
\mathcal{R}^{s} f(z)=\sum_{k=1}^{\infty} k^{s} f_{k}(z) .
$$

We define the Besov-Lipschitz space $\Lambda_{\alpha}^{p, q}\left(\mathbb{B}_{N}\right)=\Lambda_{\alpha}^{p, q}, \alpha>0$, by

$$
f \in \Lambda_{\alpha}^{p, q} \Longleftrightarrow \mathcal{R}^{s} f \in H^{p, q, s-\alpha},
$$

where $s$ is the smallest integer greater than $\alpha$. In Section 2 (Theorem 2.1) we give a new proof of the well known fact that $s$ in (1.2) can be replaced by any real number greater than $\alpha$. For $1 \leq q<\infty$ the spaces $\Lambda_{\alpha}^{p, q}$ are usually called Besov spaces. For the Lipschitz space $\Lambda_{\alpha}^{p, \infty}$ we write $\Lambda_{\alpha}^{p}=\Lambda_{\alpha}^{p, \infty}$. In particular, $\Lambda_{\alpha}^{\infty}$ consists of those $f \in H\left(\mathbb{B}_{N}\right)$ for which

$$
\sup _{z \in \mathbb{B}}\left(1-|z|^{2}\right)^{s-\alpha}\left|\mathcal{R}^{s} f(z)\right|<\infty,
$$

where $s$ is as above. This condition has sense for $s=\alpha$ as well and defines the Hardy-Sobolev spaces $H_{\alpha}^{\infty}\left(\mathbb{B}_{N}\right), \alpha>0$. More generally, the Hardy-Sobolev space $H_{\alpha}^{p}\left(\mathbb{B}_{N}\right)$ is defined by

$$
H_{\alpha}^{p}=H_{\alpha}^{p}\left(\mathbb{B}_{N}\right)=\left\{f \in H\left(\mathbb{B}_{N}\right): \mathcal{R}^{\alpha} f \in H^{p}\left(\mathbb{B}_{N}\right)\right\} .
$$

Proposition 1.1. In the definition of $\Lambda_{\alpha}^{p, q}$, condition (1.2) can be replaced by

$$
\nabla_{s}(f) \in L_{s-\alpha}^{p, q}\left(\mathbb{B}_{N}\right)
$$

where $\nabla_{s}(f)$ is the $s$-th order gradient of $f$.

\footnotetext{
${ }^{(*)}$ In [6], $\mathcal{R} f$ is defined as $f(0)+\sum_{j=1}^{N} z_{j} \partial f / \partial z_{j}$.
} 
The second order gradient is defined as

$$
\nabla_{2}(f)(z)=\left(\sum_{j, k=1}^{N}\left|\partial_{j} \partial_{k} f(z)\right|^{2}\right)^{1 / 2}
$$

where

$$
\partial_{j} f(z)=\frac{\partial f}{\partial z_{j}}(z)
$$

In the case $s>2, \nabla_{s}(f)$ is defined similarly. Of course $\nabla_{1}(f)(z)=|\nabla f(z)|$, where $\nabla f(z)$ is the ordinary holomorphic gradient.

The inequality

$$
M_{p}\left(r, \mathcal{R}^{s} f\right) \leq C \sum_{k=1}^{s-1} \nabla_{k}(f)(0)+M_{p}\left(r, \nabla_{s}(f)\right),
$$

which proves that (1.3) implies (1.2) is simple. The reverse implication can also be verified in a relatively simple way (see, e.g., the proofs of Lemmas 1 and 2 in 17]). However the following result, due to Ahern and Schneider [1, shows that much more holds.

Lemma 1.A. If $f \in H\left(\mathbb{B}_{N}\right)$, then $M_{p}(r, \nabla f) \leq C M_{p}(r, \mathcal{R} f)$, for $1 / 4<r<1$.

This fact is a reformulation of the original result of Ahern and Schneider (see Rudin [19, \$6.6.3]). We will deduce the inequality $M_{p}\left(r, \nabla_{n}(f)\right) \leq C M_{p}\left(r, \mathcal{R}^{n} f\right)$, from Lemma 1.A, in Section 4 (Lemma 4.1). In Section 4 we will also show that the radial derivative in (1.2) can also be replaced by the tangential gradients.

1.2. Mean Besov-Lipschitz spaces. Very recently, by using the $L^{p}$-modulus of continuity of order one and two, Cho, Koo and Kwon [5, 13, and Cho and Zhu 6] defined the appropriate holomorphic mean Besov-Lipschitz spaces. Their definition of the $L^{p}$-modulus of continuity can be extended as follows:

Let $\mathcal{U}$ denote the group of all unitary transformations of $\mathbb{C}^{N}$. For $U \in \mathcal{U}$ and a function $f \in H\left(\mathbb{B}_{N}\right)$, we let

$$
\begin{gathered}
f_{U}(z)=f(U z) \\
\Delta_{U}^{1} f(z)=f(U z)-f(z) \\
\Delta_{U}^{n} f=\Delta_{U}^{1}\left(\Delta_{U}^{n-1} f\right) \quad(n \geq 2) .
\end{gathered}
$$

In particular

$$
\Delta_{U}^{2} f(z)=f\left(U^{2} z\right)-2 f(U z)+f(z)
$$

Then, for $f \in H^{p}$, let

$$
\omega_{n}(\delta, f)_{p}=\sup _{\|U-I\|<\delta, U \in \mathcal{U}}\left\|\Delta_{U}^{n} f\right\|_{p} \quad(I z=z) .
$$

The holomorphic mean Besov-Lipschitz space $\operatorname{Lip}_{\alpha}^{\mathrm{p}, \mathrm{q}}\left(\mathbb{B}_{\mathrm{N}}\right)=\operatorname{Lip}_{\alpha}^{\mathrm{p}, \mathrm{q}}$ consists of those $f \in H^{p}\left(\mathbb{B}_{N}\right)$ for which

$$
\int_{0}^{1}\left[\frac{\omega_{n}(\delta, f)_{p}}{\delta^{\alpha}}\right]^{q} \frac{d \delta}{\delta}<\infty \quad \text { if } \quad 1 \leq q<\infty
$$

and

$$
\omega_{n}(\delta, f)_{p}=\mathcal{O}\left(\delta^{\alpha}\right), \quad \text { if } \quad q=\infty,
$$

where $n$ is the smallest integer greater than $\alpha$. 
We consider other two moduli:

$$
\omega_{n}^{-}(\delta, f)_{p}=\sup _{|t|<\delta}\left\|\Delta_{t}^{n} f\right\|_{p}
$$

where

$$
\Delta_{t}^{n} f=\Delta_{t}^{1}\left(\Delta_{t}^{n-1} f\right), \quad \Delta_{t}^{1} f(z)=f\left(e^{i t} z\right)-f(z), \quad t \in \mathbb{R} .
$$

To define the second one denote by $\mathcal{L}=\mathcal{L}\left(\mathbb{B}_{N}\right)$ the semigroup of all linear operators from $\mathbb{B}_{N}$ to $\mathbb{B}_{N}$, and then let

$$
\omega_{n}^{+}(\delta, f)_{p}=\sup _{\|U-I\|<\delta, U \in \mathcal{L}}\left\|\Delta_{U}^{n} f\right\|_{p}
$$

where $\Delta_{U}^{n}$ is defined as above. From (1.5), (1.6) and (1.7) we have

$$
\omega_{n}^{-}(\delta, f)_{p} \leq \omega_{n}(\delta, f)_{p} \leq \omega_{n}^{+}(\delta, f)_{p} .
$$

We are now ready to state our main result which shows that different $L^{p}$-moduli of continuity defined above give the same mean Besov-Lipschitz spaces.

Theorem 1.2. We have $\Lambda_{\alpha}^{p, q}\left(\mathbb{B}_{N}\right) \subset H^{p}\left(\mathbb{B}_{N}\right)$. For a function $f \in H^{p}$ and $0<$ $\alpha<n$, the following conditions are equivalent:

$(\mathcal{R}) f \in \Lambda_{\alpha}^{p, q}$;

$$
\begin{aligned}
(\Delta) & \left(\int_{0}^{1}\left[\frac{\left\|\Delta_{t}^{n} f\right\|_{p}}{t^{\alpha}}\right]^{q} \frac{d t}{t}\right)^{1 / q}<\infty ; \\
\left(\omega^{-}\right) & \left(\int_{0}^{1}\left[\frac{\omega_{n}^{-}(\delta, f)_{p}}{\delta^{\alpha}}\right]^{q} \frac{d \delta}{\delta}\right)^{1 / q}<\infty ; \\
(\omega) & \left(\int_{0}^{1}\left[\frac{\omega_{n}(\delta, f)_{p}}{\delta^{\alpha}}\right]^{q} \frac{d \delta}{\delta}\right)^{1 / q}<\infty ; \\
\left(\omega^{+}\right) & \left(\int_{0}^{1}\left[\frac{\omega_{n}^{+}(\delta, f)_{p}}{\delta^{\alpha}}\right]^{q} \frac{d \delta}{\delta}\right)^{1 / q}<\infty ;
\end{aligned}
$$

In the case $q=\infty$ we have

Theorem 1.3. We have $\Lambda_{\alpha}^{p}\left(\mathbb{B}_{N}\right) \subset H^{p}\left(\mathbb{B}_{N}\right)$. For a function $f \in H^{p}$ and $0<\alpha<$ $n$, the following conditions are equivalent:

$(\mathcal{R}) f \in \Lambda_{\alpha}^{p}$;

$$
\begin{aligned}
& (\Delta)\left\|\Delta_{t}^{n} f\right\|_{p}=\mathcal{O}\left(t^{\alpha}\right), 0<t<1 ; \\
& \left(\omega^{-}\right) \omega_{n}^{-}(\delta, f)_{p}=\mathcal{O}\left(\delta^{\alpha}\right), \quad 0<\delta<1 ; \\
& (\omega) \omega_{n}(\delta, f)_{p}=\mathcal{O}\left(\delta^{\alpha}\right), \quad 0<\delta<1 ; \\
& \left(\omega^{+}\right) \omega_{n}^{+}(\delta, f)_{p}=\mathcal{O}\left(\delta^{\alpha}\right), \quad 0<\delta<1 ;
\end{aligned}
$$

In the case $N=1$ Theorems 1.2 and 1.3 are known. In the case when $1 \leq p \leq \infty$, $q=\infty$ and $0<\alpha<1$, (then $\left(\omega^{-}\right) \Longleftrightarrow(\Delta)$ is clear), this theorem was proved by Hardy and Littlewood [8]. In the case $p=q=\infty, \alpha=1$, this was proved by Zygmund [21. The equivalence $(\mathcal{R}) \Longleftrightarrow\left(\omega^{-}\right)$for all $p, q, \alpha$ was proved by Oswald 
[14, while $(\mathcal{R}) \Longleftrightarrow(\Delta)$ was proved in [15, 16]. (Of course the implication $\left(\omega^{-}\right) \Longrightarrow$ $(\Delta)$ is clear.) The paper [4] of Blasco and De Souza is also relevant here.

Very recently the equivalence $(\mathcal{R}) \Longleftrightarrow(\omega)$, for $0<\alpha<2$, was proved by Cho, Koo and Kwon [5, 13, and by Cho and Zhu 6].

Concerning the Hardy-Sobolev spaces we have the following result, that was proved in [16] for $N=1$.

Theorem 1.4. Let $n$ be a positive integer. Then $H_{n}^{p}\left(\mathbb{B}_{N}\right) \subset H^{p}\left(\mathbb{B}_{N}\right)$. Moreover a function $f \in H^{p}\left(\mathbb{B}_{N}\right)$ belongs to $H_{n}^{p}\left(\mathbb{B}_{N}\right)$ if and only if

$$
\omega_{n}(\delta, f)_{p}=\mathcal{O}\left(\delta^{n}\right), \quad 0<\delta<1 .
$$

In (1.9) $\omega_{n}(\delta, f)_{p}$ may be replaced by $\omega_{n}^{-}(\delta, f)_{p}$ or by $\omega_{n}^{+}(\delta, f)_{p}$.

The implication $(\Delta) \Longrightarrow(\mathcal{R})$ in Theorem 1.2 follows from the next theorem that will be proved in Section 5. In (1.11) below just take $\psi(t)=t^{q(n-\alpha)-1}, 0<t<1$, where $0<\alpha<n$.

Theorem 1.5. Let $f \in H^{p}\left(\mathbb{B}_{N}\right)$, and let $\psi \in L^{1}(0,1)$ be a non-negative function such that

$$
\psi(2 x) \leq K \psi(x), \quad 0<x<1 / 2,
$$

where $K$ is a positive constant. Then

$$
\int_{0}^{1} M_{p}^{q}\left(r, \nabla_{n}(f)\right) \psi(1-r) d r \leq C \int_{0}^{1}\left[t^{-n}\left\|\Delta_{t}^{n} f\right\|_{p}\right]^{q} \psi(t) d t
$$

where $C$ depends only on $K, p, q, n$, and $N$.

The implication $(\Delta) \Longrightarrow(\mathcal{R})$ in Theorem 1.3 follows from the estimate

$$
M_{p}\left(r, \nabla_{n}(f)\right) \leq C(1-r)^{-n-1} \int_{0}^{1-r}\left\|\Delta_{t}^{n} f\right\|_{p} d t
$$

which is a consequence of Theorem 1.5. (See Section 5).

The implications $\left(\omega^{+}\right) \Longrightarrow(\omega) \Longrightarrow\left(\omega^{-}\right) \Longrightarrow(\Delta)$ follow from (1.8) and the definition of $\left(\omega^{-}\right)$. To finish the proofs of Theorem 1.2 and Theorem 1.3 it remains to prove the implication $(\mathcal{R}) \Longrightarrow\left(\omega^{+}\right)$. This follows from the following theorem.

Theorem 1.6. If $f \in H\left(\mathbb{B}_{N}\right)$ and

$$
\int_{0}^{1}(1-r)^{n-1} M_{p}\left(r, \mathcal{R}^{n} f\right) d r<\infty
$$

then $f \in H^{p}$, and

$$
\omega_{n}^{+}(\delta, f)_{p} \leq C \int_{1-\delta}^{1} M_{p}\left(r, \mathcal{R}^{n} f\right)(1-r)^{n-1} d r, \quad 0<\delta<1 .
$$

The proof that (1.13) implies $f \in H^{p}$ will be given in Section 2 (Corollary 2.5), and the proof of (1.14) will be given in Section 5 .

In Section 2 (Corollary 2.4) we prove that $\Lambda_{\alpha}^{p, q} \subset H^{p}$. Since the condition $f \in H_{n}^{p}$ implies (1.13), we have $H_{n}^{p} \subset H^{p}$. To finish the proof of Theorem 1.4 apply (1.12) and (1.14).

In the case $N=1$, Theorem 1.6 was proved in [16. Theorem 2.2]. Similar theorems appear in $[5$ in the case where $n=1,2$. For example, Theorems 5.2 and 
5.3 of [5] give

$$
\omega_{2}(\delta, f)_{p} \leq C \delta^{2} M_{p}\left(1-\delta, \mathcal{R}^{2} f\right)+C \int_{1-\delta}^{1}(1-r) M_{p}\left(r, \mathcal{R}^{2} f\right) d r .
$$

Here we observe that the summand $\delta^{2} M_{p}\left(1-\delta, \mathcal{R}^{2} f\right)$ is not needed because, by the increasing property of the integral means,

$$
\delta^{2} M_{p}\left(1-\delta, \mathcal{R}^{2} f\right) \leq 2 \int_{1-\delta}^{1}(1-r) M_{p}\left(r, \mathcal{R}^{2} f\right) d r .
$$

As a consequence we note a generalization of [5, Theorem 5.3]:

Corollary 1.7. We have

$$
\omega_{n}^{+}\left(\delta, f_{1-\delta}\right)_{p} \leq C \delta^{n} M_{p}\left(1-\delta, \mathcal{R}^{n} f\right), \quad 0<\delta<1 .
$$

Proof. It follows from the Theorem 1.6 (relation (1.14)), applied to the function $f_{\rho}(z)=f(\rho z)$, and the increasing property of the integral means that

$$
\omega_{n}^{+}\left(\delta, f_{\rho}\right)_{p} \leq C \delta^{n} M_{p}\left(\rho, \mathcal{R}^{n} f\right), \quad 0<\rho<1,0<\delta<1 .
$$

Now (1.15) is obtained by taking $\rho=1-\delta$.

Remark 1.8. We will use a weaker variant of (1.15) in the proof of Theorem 1.6 (see Lemma 5.5).

In order to give a further consequence of Theorem 1.6, let $\phi>0$ be an continuous increasing function on the interval $(0,1]$ and define the spaces $\Lambda_{n, \phi}^{p}\left(\mathbb{B}_{N}\right)$ and $\operatorname{Lip}_{n, \phi}^{p}\left(\mathbb{B}_{N}\right)$ in the following way:

The space $\Lambda_{n, \phi}^{p}\left(\mathbb{B}_{N}\right)$ consists of those $f \in H\left(\mathbb{B}_{N}\right)$ for which

$$
M_{p}\left(r, \mathcal{R}^{n} f\right)=\mathcal{O}\left(\frac{\phi(1-r)}{(1-r)^{n}}\right), \quad r \rightarrow 1^{-} .
$$

The space $\operatorname{Lip}_{n, \phi}^{p}\left(\mathbb{B}_{N}\right)$ consists of those $f \in H^{p}\left(\mathbb{B}_{N}\right)$ for which

$$
\omega_{n}^{+}(\delta, f)_{p}=\mathcal{O}(\phi(\delta)), \quad \delta \rightarrow 0^{+} .
$$

The little "oh" spaces $\lambda_{n, \phi}^{p}$ and lip ${ }_{n, \phi}^{p}$ are defined by replacing " $\mathcal{O}$ " with "o". The condition $t^{n}=\mathcal{O}(\phi(t))$ guarantees that the space $\Lambda_{n, \phi}^{p}$ is of infinite dimension; in fact then $\Lambda_{n, \phi}^{p}$ contains the Hardy-Sobolev space $H_{n}^{p}\left(\mathbb{B}_{N}\right)$. This condition should be strengthened to $t^{n}=o(\phi(t)), t \rightarrow 0^{+}$, if we want $\lambda_{n, \phi}^{p}\left(\mathbb{B}_{N}\right)$ to be infinitedimensional.

Corollary 1.9. If the function $x \mapsto \phi(x) / x^{\alpha}$ increases on $(0,1]$ for some $0<\alpha<n$, then $\Lambda_{n, \phi}^{p}\left(\mathbb{B}_{N}\right) \subset \operatorname{Lip}_{n, \phi}^{p}\left(\mathbb{B}_{N}\right)$ and $\lambda_{n, \phi}^{p}\left(\mathbb{B}_{N}\right) \subset \operatorname{lip}_{n, \phi}^{p}\left(\mathbb{B}_{N}\right)$.

Proof. If $f \in \Lambda_{n, \phi}^{p}$, then $f \in \Lambda_{\alpha}^{p}$ (because $\left.\phi(t) \leq \phi(1) t^{\alpha}\right)$ and hence $f \in H^{p}\left(\mathbb{B}_{N}\right)$ (see Corollary 2.4). Next,

$$
\begin{aligned}
\omega_{n}^{+}(\delta, f)_{p} & \leq C \int_{0}^{\delta} \frac{\phi(x)}{x} d x \\
& =C \int_{0}^{\delta} \frac{\phi(x)}{x^{\alpha}} x^{\alpha-1} d x \\
& \leq C \frac{\phi(\delta)}{\delta^{\alpha}} \frac{\delta^{\alpha}}{\alpha}
\end{aligned}
$$


and hence $f \in \operatorname{Lip}_{n, \phi}^{p}$. The rest is proved similarly.

Note that $\operatorname{Lip}_{n, \phi}^{p}\left(\mathbb{B}_{N}\right) \subset \Lambda_{n, \phi}^{p}\left(\mathbb{B}_{N}\right)$. Use (1.12) and (1.16).

The above corollary can be generalized to integrated Besov-Lipschitz spaces. Let $\Lambda_{n, \phi}^{p, q}\left(\mathbb{B}_{N}\right), 1 \leq q<\infty$, denote the class of those $f \in H\left(\mathbb{B}_{N}\right)$ for which

$$
\int_{0}^{1}\left(M_{p}\left(r, \mathcal{R}^{n} f\right) \frac{(1-r)^{n}}{\phi(1-r)}\right)^{q} \frac{d r}{1-r}<\infty .
$$

The space $\operatorname{Lip}_{n, \phi}^{p, q}\left(\mathbb{B}_{N}\right)$ is the subclass of $H^{p}\left(\mathbb{B}_{N}\right)$ for which

$$
\int_{0}^{1}\left(\frac{\left\|\Delta_{t}^{n} f\right\|_{p}}{\phi(t)}\right)^{q} \frac{d t}{t}<\infty
$$

Suppose

$$
\int_{0}^{1}\left(\frac{t^{n}}{\phi(t)}\right)^{q} \frac{d t}{t}<\infty
$$

which implies that $\Lambda_{n, \phi}^{p, q}\left(\mathbb{B}_{N}\right)$ is infinite-dimensional (in fact, it contains all polynomials). If $(1.20)$ is satisfied then $\operatorname{Lip}_{n, \phi}^{p, q}\left(\mathbb{B}_{N}\right) \subset \Lambda_{n, \phi}^{p, q}\left(\mathbb{B}_{N}\right)$. This fact is an easy consequence of Theorem 1.5. (Use (1.11),(1.18) and (1.19).)

Corollary 1.10. If the function $x \mapsto \phi(x) / x^{\alpha}$ increases on $(0,1]$ for some $\alpha>0$, then $\Lambda_{n, \phi}^{p, q}\left(\mathbb{B}_{N}\right) \subset \operatorname{Lip}_{n, \phi}^{p, q}\left(\mathbb{B}_{N}\right)$.

Proof. Since $\phi(t) \leq \phi(1) t^{\alpha}$, we have $\Lambda_{n, \phi}^{p, q} \subset \Lambda_{\alpha}^{p, q} \subset H^{p}$.

Let $f \in \Lambda_{n, \phi}^{p, q}$. We prove that $f \in \operatorname{Lip}_{n, \phi}^{p, q}$. By Jensen's inequality,

$$
\left(\int_{1-t}^{1}(1-r)^{n-1} M_{p}\left(r, \mathcal{R}^{n} f\right) \frac{\alpha d r}{t^{\alpha}}\right)^{q} \leq \int_{1-t}^{1}(1-r)^{q(n-\alpha)+\alpha-1} M_{p}\left(r, \mathcal{R}^{n} f\right)^{q} \frac{\alpha d r}{t^{\alpha}} .
$$

From this and (1.14) we have

$$
\begin{aligned}
& \int_{0}^{1}\left(\frac{\left\|\Delta_{t}^{n} f\right\|_{p}}{\phi(t)}\right)^{q} \frac{d t}{t} \leq \int_{0}^{1}\left(\frac{\omega_{n}^{+}(t, f)_{p}}{\phi(t)}\right)^{q} \frac{d t}{t} \\
& \leq C \int_{0}^{1} \frac{t^{\alpha q-\alpha-1}}{(\phi(t))^{q}}\left(\int_{1-t}^{1}(1-r)^{q(n-\alpha)+\alpha-1} M_{p}\left(r, \mathcal{R}^{n} f\right)^{q} d r\right) d t \\
& =C \int_{0}^{1}(1-r)^{q(n-\alpha)+\alpha-1} M_{p}\left(r, \mathcal{R}^{n} f\right)^{q}\left(\int_{1-r}^{1} \frac{t^{\alpha q-\alpha-1}}{\phi(t)^{q}} d t\right) d r
\end{aligned}
$$

Using the inequality $\frac{t^{\alpha}}{\phi(t)} \leq \frac{(1-r)^{\alpha}}{\phi(1-r)}, 1-r \leq t$, one shows that the inner integral is dominated by $\frac{C(1-r)^{\alpha q-\alpha}}{(\phi(1-r))^{q}}$, which completes the proof.

\section{Finite-dimensional DeCOMPosition And applications}

Let $\psi:(0, \infty) \mapsto \mathbb{C}$ be a $C^{\infty}$ function with compact support in $(0, \infty)$. Consider the polynomials

$$
\Omega_{\nu}(z)=\Omega_{\nu}^{\psi}(z)=\sum_{j=0}^{\infty} \psi\left(\frac{j}{2^{\nu-1}}\right) z^{j}, \quad z \in \mathbb{B}_{1}, \quad \nu \geq 1 .
$$

It is proved in [18, Theorem 7.3.4] that

$$
\left\|\Omega_{\nu} * f\right\|_{p} \leq C\|f\|_{p}, \quad f \in H^{p}\left(\mathbb{B}_{N}\right),
$$


in the case $N=1$. (Theorem 7.3.4 of [18] says much more than we need. Inequality (2.1) is rather elementary, see [18, Lemma 7.3.2] or [10.) The Hadamard product of a functions $f \in H^{p}\left(\mathbb{B}_{N}\right)$ and $g \in H^{p}\left(\mathbb{B}_{1}\right)$ is defined as

$$
g * f(z)=\sum_{k=0}^{\infty} \hat{g}(k) f_{k}(z)
$$

where $f=\sum_{k=0}^{\infty} f_{k}$ is the homogeneous expansion of $f$.

Integration by slices shows that (2.1) holds for all $N$. Using this one can choose $\psi(t)=\omega(t / 2)-\omega(t)$, where $\omega(t)$ is any infinitely differentiable function with $\omega(t)=$ 1 for $t \leq 1,0 \leq \omega(t) \leq 1$ for $1<t \leq 2$ and $\omega(t)=0$ for $t>2$, so that the polynomials $\Omega_{\nu}^{\psi}=: W_{\nu}$ satisfies:

$$
\begin{gathered}
\operatorname{supp} \widehat{W}_{\nu} \subset\left[2^{\nu-1}, 2^{\nu+1}\right), \quad \nu \geq 1, \\
f(z)=\sum_{\nu=0}^{\infty} W_{\nu} * f(z), \quad f \in H\left(\mathbb{B}_{N}\right),
\end{gathered}
$$

where

$$
W_{0}(z)=1+z,
$$

and

$$
\left\|W_{\nu} * f\right\|_{p} \leq C\|f\|_{p}, \quad f \in H\left(\mathbb{B}_{N}\right) .
$$

(See, e.g. 11.)

We define $\mathcal{B}_{\beta}^{p, q}=\mathcal{B}_{\beta}^{p, q}\left(\mathbb{B}_{N}\right),-\infty<\beta<\infty$, to be the space of those $f \in H\left(\mathbb{B}_{N}\right)$ for which

$$
\|f\|_{\mathcal{B}_{\beta}^{p, q}}:=\left\|\left\{2^{-\nu \beta}\left\|W_{\nu} * f\right\|_{p}\right\}_{\nu=0}^{\infty}\right\|_{\ell^{q}}<\infty .
$$

The following theorem, for $N=1$, is proved in [12]. A similar argument shows that it holds for all $N \geq 1$. See [11, Lemma 2.2].

Theorem 2.A. We have $H^{p, q, \alpha}\left(\mathbb{B}_{N}\right)=\mathcal{B}_{\alpha}^{p, q}\left(\mathbb{B}_{N}\right)$.

Theorem 2.1. We have $\Lambda_{\alpha}^{p, q}=\mathcal{B}_{-\alpha}^{p, q}$. More generally, if $s>\alpha$ is any real number, then

$$
\left\{f \in H\left(\mathbb{B}_{N}\right):\left\|\mathcal{R}^{s} f\right\|_{p, q, s-\alpha}<\infty\right\}=\mathcal{B}_{-\alpha}^{p, q},
$$

and consequently

$$
\Lambda_{\alpha}^{p, q}=\left\{f \in H\left(\mathbb{B}_{N}\right):\left\|\mathcal{R}^{s} f\right\|_{p, q, s-\alpha}<\infty\right\} .
$$

Remark 2.2. Relation (2.5) is proved in [6, Theorem 3.11] in a different manner.

Proof. This is an immediate consequence of Theorem 2.A and the following lemma.

Lemma 2.3. If $f \in H\left(\mathbb{B}_{N}\right)$, and $s \in \mathbb{C}$, then

$$
\left\|W_{\nu} * \mathcal{R}^{s} f\right\|_{p} \asymp 2^{\nu \operatorname{Re} s}\left\|W_{\nu} * f\right\|_{p}, \quad \nu \geq 2 .
$$

The notation $a \asymp b$ means that $a / b$ lies between two positive constants. In this case these constants are independent of $\nu$ and $f$. 
Proof. Let

$$
Q_{\nu}=W_{\nu-1}+W_{\nu}+W_{\nu+1}, \quad \nu \geq 0,
$$

where $W_{-1}:=0$. Since $Q_{\nu} * W_{k}=0$ for $|\nu-k| \geq 2$, it follows from (2.2) that

$$
Q_{\nu} * W_{\nu}=W_{\nu} .
$$

Let

$$
\varphi_{s}(x)=x^{s}(\psi(x / 2)+\psi(x)+\psi(2 x)) .
$$

We have, for $\nu \geq 2$,

$$
Q_{\nu} * \mathcal{R}^{s} f=2^{(\nu-1) s} \Omega_{\nu}^{\varphi_{s}} * f .
$$

From this and (2.1) we get

$$
\left\|Q_{\nu} * \mathcal{R}^{s} f\right\|_{p} \leq C 2^{\nu \operatorname{Re} s}\|f\|_{p}
$$

Replacing $f$ by $W_{\nu} * f$ we get

$$
\begin{aligned}
\left\|W_{\nu} * \mathcal{R}^{s} f\right\|_{p} & =\left\|Q_{\nu} * \mathcal{R}^{s}\left(W_{\nu} * f\right)\right\|_{p} \\
& \leq C 2^{\nu \operatorname{Re} s}\left\|W_{\nu} * f\right\|_{p}
\end{aligned}
$$

The reverse inequality now follows from the relation

$$
W_{\nu} * f=W_{\nu} * \mathcal{R}^{-s}\left(\mathcal{R}^{s} f\right) .
$$

Corollary 2.4. We have $\Lambda_{\alpha}^{p, q}\left(\mathbb{B}_{N}\right) \subset H^{p}\left(\mathbb{B}_{N}\right)$.

Proof. Let $f \in \Lambda_{\alpha}^{p, q}\left(\mathbb{B}_{N}\right)$. Then, by Theorem 2.1, $\left\|W_{\nu} * f\right\|_{p} \leq C 2^{-n \alpha}$. It follows that

$$
\|f\|_{p} \leq \sum_{\nu=0}^{\infty}\left\|W_{\nu} * f\right\|_{p}<\infty .
$$

Corollary 2.5. If $f \in H\left(\mathbb{B}_{N}\right)$ and

$$
K:=\int_{0}^{1}(1-r)^{n-1} M_{p}\left(r, \mathcal{R}^{n} f\right) d r<\infty,
$$

then $f \in H^{p}$, and $\|f\|_{p} \leq C\left(|f(0)|+K^{1 / p}\right)$.

Proof. by Theorem 2.A and Lemma 2.3, we have

$$
K+|f(0)| \asymp \sum_{\nu=0}^{\infty}\left\|W_{\nu} * f\right\|_{p} .
$$

The result follows. 
2.1. The operators $\mathcal{R}^{s, t}$. Let $s, t$ be real numbers such that neither $N+s$ nor $N+s+t$ is a negative integer. Let

$$
\mathcal{R}^{s, t} f(z)=\sum_{k=0}^{\infty} \frac{\Gamma(N+1+s) \Gamma(N+1+k+s+t)}{\Gamma(N+1+s+t) \Gamma(N+1+k+s)} f_{k}(z) .
$$

The following theorem is proved in [6, Theorem 3.10].

Theorem 2.B. Suppose $t>\alpha, f \in H^{p}$. If $s$ is a real number such that neither $N+s$ nor $N+s+t$ is a negative integer, then $f \in \Lambda_{\alpha}^{p, q}$ if and only if $\mathcal{R}^{s, t} f \in L_{t-\alpha}^{p, q}$.

An application of Stirling's formula shows that

$$
\frac{\Gamma(N+1+s) \Gamma(N+1+k+s+t)}{\Gamma(N+1+s+t) \Gamma(N+1+k+s)}=k^{t}\left(a_{1}+\frac{a_{2}}{k}+\mathcal{O}\left(\frac{1}{k^{2}}\right)\right), \quad k \rightarrow \infty,
$$

where $a_{1} \neq 0$ and $a_{2}$ are constants. Therefore Theorem 2.B is a consequence of the following result.

Theorem 2.6. Let $T: H\left(\mathbb{B}_{N}\right) \mapsto H\left(\mathbb{B}_{N}\right)$ be an operator of the form

$$
T f(z)=\sum_{k=0}^{\infty} \lambda_{k} f_{k}(z)
$$

where

$$
\lambda_{k}=k^{t}\left(a_{1}+\frac{a_{2}}{k}+o\left(\frac{1}{k}\right)\right), \quad k \rightarrow \infty,
$$

and $t, a_{1} \neq 0$, and $a_{2}$ are constants. Let $t>\alpha$. Then $f \in \Lambda_{\alpha}^{p, q}$ if and only if $T f \in L_{t-\alpha}^{p, q}$.

Proof. By Theorem 2.A and Lemma 2.3, it suffices to prove that

$$
\left\|T\left(W_{\nu} * f\right)\right\|_{p} \asymp\left\|W_{\nu} * f\right\|_{p}, \quad \nu \rightarrow \infty,
$$

under the hypothesis $t=0$ and $a_{1}=1$. Let $Q=W_{\nu} * f$. Let

$$
T_{1} f(z)=\sum_{k=1}^{\infty} \frac{\eta_{k}}{k} f_{k}(z)
$$

where $\eta_{k} \rightarrow 0$ as $k \rightarrow \infty$. Let $Q_{k}(z)=\widehat{W}_{\nu}(k) f_{k}(z)$. Then

$$
T Q=Q+a_{2} \mathcal{R}^{-1} Q+T_{1} Q .
$$

Hence, by Lemma 2.3 and the inequality $\left\|Q_{k}\right\|_{p} \leq\|Q\|_{p}$,

$$
\begin{aligned}
\|T Q\|_{p} & \leq\|Q\|_{p}+C_{1}\left|a_{2}\right| 2^{-\nu}\|Q\|_{p}+2^{1-\nu} \sum_{k=2^{\nu-1}}^{2^{\nu+1}}\left|\eta_{k}\right|\left\|Q_{k}\right\|_{p} \\
& \leq\|Q\|_{p}+C_{1}\left|a_{2}\right| 2^{-\nu}\|Q\|_{p}+2^{1-\nu}\|Q\|_{p} \sum_{k=2^{\nu-1}}^{2^{\nu+1}}\left|\eta_{k}\right| \\
& \leq C\|Q\|_{p} .
\end{aligned}
$$


In the other direction we have

$$
\begin{aligned}
\|T Q\|_{p} & \geq\|Q\|_{p}-C_{1}\left|a_{2}\right| 2^{-\nu}\|Q\|_{p}-2^{1-\nu} \sum_{k=2^{\nu-1}}^{2^{\nu+1}}\left|\eta_{k}\right|\left\|Q_{k}\right\|_{p} \\
& \geq\|Q\|_{p}-C_{1}\left|a_{2}\right| 2^{-\nu}\|Q\|_{p}-2^{1-\nu}\|Q\|_{p} \sum_{k=2^{\nu-1}}^{2^{\nu+1}}\left|\eta_{k}\right|
\end{aligned}
$$

Now choose $\nu_{0}$ so that $C_{1}\left|a_{2}\right| 2^{-\nu}<1 / 4$ and $2^{1-\nu} \sum_{k=2^{\nu-1}}^{2^{\nu+1}}\left|\eta_{k}\right|<1 / 4$ for $\nu>\nu_{0}$ to get $\|T Q\|_{p} \geq(1 / 2)\|Q\|_{p}$. This proves the theorem.

2.2. The case $1<p<\infty$. In this case the above discussion can be made simpler by using the Riesz projection theorem. Namely, then we can replace $W_{\nu}$ by

$$
V_{\nu}(z)=\sum_{k=2^{\nu-1}}^{2^{\nu}-1} z^{k}, \quad z \in \mathbb{B}_{1}, \nu \geq 1,
$$

and $V_{0}=1$. Obviously, these polynomials satisfy (2.2) and also, by the projection theorem, (2.3) $(1<p<\infty)$.

\section{BeSt APPROXIMATION BY POLYNOMIALS}

For a function $f \in H^{p}\left(\mathbb{B}_{N}\right)$, let

$$
E_{\nu}(f)_{p}=\inf \left\{\|f-P\|_{p}: P \in \mathscr{P}_{\nu}\left(\mathbb{B}_{N}\right)\right\},
$$

where $\mathscr{P}_{\nu}\left(\mathbb{B}_{N}\right)$ is the subset of $H\left(\mathbb{B}_{N}\right)$ consisting of all polynomials of degree $\leq \nu$. The following characterization of the one variable Besov-Lipschitz spaces is well known (for a proof see [9]).

Theorem 3.1. A function $f \in H^{p}\left(\mathbb{B}_{N}\right)$ is in $\Lambda_{\alpha}^{p, q}$ if and only if the sequence $\left\{2^{\nu \alpha} E_{2^{\nu}}(f)_{p}\right\}_{\nu=0}^{\infty}$ is in $\ell^{q}$.

Proof. Let $P_{\nu}$ be a sequence of polynomials of degree $\leq 2^{\nu}$ such that

$$
\left\{2^{\nu \alpha}\left\|f-P_{\nu}\right\|_{p}\right\}_{0}^{\infty} \in \ell^{q} .
$$

Since

$$
\left(f-P_{\nu}\right) * W_{\nu+2}=f * W_{\nu+2}
$$

we have

$$
\left\|W_{\nu+2} * f\right\|_{p} \leq C\left\|f-P_{\nu}\right\|_{p} .
$$

This and Theorem 2.1 show that $f \in \Lambda_{\alpha}^{p, q}$. To prove the converse observe that $Q_{\nu}=\sum_{k=0}^{\nu-1} W_{k}, \nu \geq 1$, is a polynomial of degree $\leq 2^{\nu}$, and therefore by using (2.2) we obtain

$$
\begin{aligned}
E_{2^{\nu}}(f)_{p} & \leq\left\|f-Q_{\nu} * f\right\|_{p} \\
& \leq \sum_{k=\nu-1}^{\infty}\left\|W_{k} * f\right\|_{p} .
\end{aligned}
$$

Hence

$$
\left\|\left\{2^{\nu \alpha} E_{2^{\nu}}(f)_{p}\right\}_{\nu=0}^{\infty}\right\|_{\ell^{q}} \leq\left\|\left\{2^{\nu \alpha} s_{\nu-1}\right\}_{\nu=1}^{\infty}\right\|_{\ell^{q}},
$$

where

$$
s_{\nu}=\sum_{k=\nu}^{\infty}\left\|W_{k} * f\right\|_{p} .
$$


MIROLJUB JEVTIĆ AND MIROSLAV PAVLOVIĆ

Now the desired result follows Theorem 2.1 and the following lemma.

Lemma 3.2. If $\left\{s_{\nu}\right\}_{0}^{\infty}$ is a sequence of complex numbers such that $\left\{2^{\nu \alpha} \mid s_{\nu}-\right.$ $\left.s_{\nu-1} \mid\right\}_{1}^{\infty} \in \ell^{q}$, then $\left\{2^{\nu \alpha}\left|s_{\nu}\right|\right\}_{1}^{\infty} \in \ell^{q}$.

Proof. Assuming, as we may, $s_{\nu}$ is eventually zero, we have

$$
\begin{aligned}
M:=\left\|\left\{2^{\nu \alpha} s_{\nu-1}\right\}_{\nu=1}^{\infty}\right\|_{\ell^{q}} & \leq\left\|\left\{2^{\nu \alpha}\left|s_{\nu-1}-s_{\nu}\right|\right\}_{\nu=1}^{\infty}\right\|_{\ell^{q}}+\left\|\left\{2^{\nu \alpha} s_{\nu}\right\}_{\nu=1}^{\infty}\right\|_{\ell^{q}} \\
& =\left\|\left\{2^{\nu \alpha}\left|s_{\nu-1}-s_{\nu}\right|\right\}_{\nu=1}^{\infty}\right\|_{\ell^{q}}+\left\|\left\{2^{(\nu-1) \alpha} s_{\nu-1}\right\}_{\nu=2}^{\infty}\right\|_{\ell^{q}} \\
& \leq\left\|\left\{2^{\nu \alpha}\left|s_{\nu-1}-s_{\nu}\right|\right\}_{\nu=1}^{\infty}\right\|_{\ell^{q}}+2^{-\alpha}\left\|\left\{2^{\nu \alpha} s_{\nu-1}\right\}_{\nu=2}^{\infty}\right\|_{\ell^{q}} \\
& \leq\left\|\left\{2^{\nu \alpha}\left|s_{\nu-1}-s_{\nu}\right|\right\}_{\nu=1}^{\infty}\right\|_{\ell^{q}}+2^{-\alpha} M .
\end{aligned}
$$

Since $M$ is finite we get

$$
M \leq\left(1-2^{-\alpha}\right)^{-1} \|\left\{2^{\nu \alpha}\left|s_{\nu-1}-s_{\nu}\right|_{\nu=1}^{\infty} \|_{\ell^{q}} .\right.
$$

This proves the lemma.

\section{Characterizations of Besov-Lipschitz spaces}

Note that another way to express $\mathcal{R}$ is

$$
\mathcal{R} f\left(r e^{i \theta} \zeta\right)=-i \frac{\partial}{\partial \theta} f\left(r e^{i \theta} \zeta\right), \quad|\zeta|=1,0 \leq r<1 .
$$

As it was noticed in the introduction Proposition 1.1 follows from the inequality (1.4) and the following inequality:

Lemma 4.1. $\left\|\nabla_{n}(f)\right\|_{p} \leq C\left\|\mathcal{R}^{n} f\right\|_{p}$

Proof. In the case $n=1$ this is just Lemma 1 .A. Let $n \geq 2$. We have to prove that

$$
\left\|\partial_{j_{1}} \partial_{j_{2}} \ldots \partial_{j_{n}} f\right\|_{p} \leq C\left\|\mathcal{R}^{n} f\right\|_{p}
$$

where $1 \leq j_{1}, j_{2}, \ldots, j_{n} \leq N$. By induction hypothesis, we have

$$
\left\|\partial_{j_{1}} \partial_{j_{2}} \ldots \partial_{j_{n}} f\right\|_{p} \leq C\left\|\mathcal{R}^{n-1} \partial_{j_{n}} f\right\|_{p}
$$

On the other hand, it is easy to see (by induction) that

$$
\mathcal{R}^{n-1} \partial_{j} f=\partial_{j}(\mathcal{R}-I)^{n-1} f
$$

whence, by Lemma 1.A,

$$
\begin{aligned}
\left\|\mathcal{R}^{n-1} \partial_{j_{n}} f\right\|_{p} & \leq \sum_{k=0}^{n-1}\left(\begin{array}{c}
n-1 \\
k
\end{array}\right)\left\|\partial_{j_{n}} \mathcal{R}^{k} f\right\|_{p} \\
& \leq C \sum_{k=0}^{n-1}\left(\begin{array}{c}
n-1 \\
k
\end{array}\right)\left\|\mathcal{R}^{k+1} f\right\|_{p}
\end{aligned}
$$

Now we prove that

$$
\|\mathcal{R} f\|_{p} \leq\left\|\mathcal{R}^{2} f\right\|_{p}
$$

which will conclude the proof. First observe that (4.3) reduces to the case $N=1$ by using integration by slices. We also may assume that $f$ is a polynomial. Then

Hence

$$
\mathcal{R} f(\zeta)=\int_{0}^{1} \frac{1}{r} \mathcal{R}^{2} f(r \zeta) d r, \quad|\zeta|=1 .
$$

$$
\|\mathcal{R} f\|_{p} \leq \int_{0}^{1} \frac{1}{r} M_{p}\left(r, \mathcal{R}^{2} f\right) d r=\int_{0}^{1} M_{p}(r, g) d r
$$


where

$$
g(r \zeta)=\sum_{k=1}^{\infty} k^{2} \hat{f}(k) r^{k-1} \zeta^{k-1}
$$

Since

$$
M_{p}(r, g) \leq\|g\|_{p}=\left\|\mathcal{R}^{2} f\right\|_{p}
$$

the proof of (4.3) and of the lemma is completed.

For the characterizations of Besov-Lipschitz spaces that will be given below we use tangential derivatives. For $1 \leq i, j \leq N$ the tangential derivatives $T_{i, j}$ and $\bar{T}_{i, j}$ are defined by

$$
T_{i, j}=\bar{z}_{i} \frac{\partial}{\partial z_{j}}-\bar{z}_{j} \frac{\partial}{\partial z_{i}} \quad \text { and } \quad \bar{T}_{i, j}=z_{i} \frac{\partial}{\partial \bar{z}_{j}}-z_{j} \frac{\partial}{\partial \bar{z}_{i}} .
$$

We will consider operators $T=T_{1} \cdots T_{k}$ where the $T_{1}, \ldots, T_{k}$ are chosen among either the $T_{i, j}$ or the $\bar{T}_{i, j}, 1 \leq i, j \leq N$. We define by $\left\{T_{\delta}^{+}\right\}, \delta \in C_{k}^{+}$the collection of such operators and define

$$
\nabla_{T^{+}}^{k} f(z)=\sum_{\delta \in C_{k}^{+}}\left|T_{\delta}^{+} f(z)\right|
$$

If $T_{1}, \ldots, T_{k}$ are chosen among the $T_{i, j}$ (not the $\bar{T}_{i, j}$ 's ), the collection of such $T=T_{1} \cdots T_{k}$ will be denoted by $\left\{T_{\delta}\right\}, \delta \in C_{k}$ and the complex-tangential gradient as

$$
\nabla_{T}^{k} f(z)=\sum_{\delta \in C_{k}}\left|T_{\delta} f(z)\right|
$$

We recall the definition of the non-isotropic weight of a differential operator. We assign weight 1 to $\mathcal{R}$ while $T_{i, j}$ and $\bar{T}_{i, j}$ are given weight $1 / 2$ each. We will consider differential operators

$$
X f=X_{1} \cdots X_{k} f
$$

where each $X_{j}$ is $\mathcal{R}$ or the one of $T_{i, j}$ or $\bar{T}_{i, j}$. For such an operator its weight is defined to be the sum of each weights of $X_{j}$.

For $z \in \mathbb{B}_{N}$ and $\delta>0$ let $P(z, \delta)$ be the non-isotropic polydisc defined as follows. If $z=r \xi, 0 \leq r<1, \xi \in S_{N}$, pick $\eta_{2}, \ldots, \eta_{N}$ so that $\left\{\xi, \eta_{2}, \ldots, \eta_{N}\right\}$ is an orthonormal basis of $\mathbb{C}^{N}$. Then

$$
P(z, \delta)=\left\{w=r \xi+\lambda \xi+\sum_{j=2}^{N} \lambda_{j} \eta_{j}, \quad|\lambda|<\delta,\left|\lambda_{j}\right|<\sqrt{\delta}, j=2,3, \ldots, N\right\}
$$

The following is a weak version of Lemma 2.5([2]).

Lemma 4.2. Let $X$ and $Y$ be the differential operators of the form (4.4) with weight of $X$ being $m$. If $f \in H\left(\mathbb{B}_{N}\right)$, then we have

$$
|X Y f(z)|^{p} \leq \frac{C}{\delta^{N+1+m p}} \int_{P(z, \delta)}|Y f(w)|^{p} d v(w),
$$

for $P(z, \delta) \subset \mathbb{B}_{N}$.

As a corollary we have that

$$
M_{p}(r, X Y f) \leq \frac{C}{(1-r)^{m}} M_{p}(\bar{r}, Y f),
$$

where $1 / 2<r<1$ and $\bar{r}=r+\frac{1-r}{4}$. 
We will also need the following two lemmas.

Lemma 4.3. ([2]) Let $\alpha>0, \beta>0$ and $1 \leq p<\infty$. Then we have

$$
\int_{0}^{1}(1-r)^{\alpha-1}\left(\int_{0}^{r}(r-t)^{\beta-1} F(t) d t\right)^{p} d r \leq C \int_{0}^{1}(1-r)^{\alpha+\beta p-1} F(r)^{p} d r
$$

for all $F \geq 0$.

Lemma 4.4. ([5]) Let $m$ and $k$ be positive integers. If $f \in H\left(\mathbb{B}_{N}\right)$, then for $T_{\delta}^{+} f$, $\delta \in C_{k}^{+}$, and $1 / 2<r<1$, we have

$$
M_{p}\left(r, T_{\delta}^{+} f\right) \leq C\left(\sup _{|z|<1 / 2}|f(z)|+\int_{0}^{r}(r-t)^{m-1} M_{p}\left(t, T_{\delta}^{+} \mathcal{R}^{m} f\right) d t\right) .
$$

Now we are ready to give the characterization of Besov-Lipschitz spaces that involve the tangential derivatives.

Theorem 4.5. Suppose that $k>2 \alpha, k$ is an integer. If $f \in H^{p}$ then the following statements are equivalent:

(i) $f \in \Lambda_{\alpha}^{p, q}$;

(ii) $\left\|\nabla_{T}^{k} f\right\|_{p, q, k / 2-\alpha}<\infty$;

(iii) $\left\|\nabla_{T^{+}}^{k} f\right\|_{p, q, k / 2-\alpha}<\infty$.

The equivalence $(i) \Longleftrightarrow($ iii $)$, for $0<\alpha<2$, was considered in [5, 13].

Proof. Obviously, (iii) $\Rightarrow(i i)$. Now we show that, for $1 \leq q<\infty,(i) \Rightarrow($ iii $)$. Let $f \in \Lambda_{\alpha}^{p, q}$. Assume that $m$ is a positive integer such that $0<\alpha<m$. By Theorem 2.1

$$
\int_{0}^{1}(1-r)^{q(m-\alpha)-1} M_{p}\left(r, \mathcal{R}^{m} f\right)^{q} d r<\infty .
$$

By using Lemma 4.4, (4.6) and (4.5) we find for $\delta \in C_{k}^{+}$

$$
\begin{aligned}
& \left\|T_{\delta}^{+} f\right\|_{p, q, k / 2-\alpha} \leq C\left(\sup _{|z|<1 / 2}|f(z)|\right. \\
& \left.+\left(\int_{0}^{1}(1-r)^{q(k / 2-\alpha)-1}\left(\int_{0}^{r}(r-t)^{m-1} M_{p}\left(t, T_{\delta}^{+} \mathcal{R}^{m} f\right)^{q} d t\right)^{q} d r\right)^{1 / q}\right) \\
& \leq C\left(\sup _{|z|<1 / 2}|f(z)|+\left(\int_{0}^{1}(1-r)^{q(k / 2+m-\alpha)-1} M_{p}\left(r, T_{\delta}^{+} \mathcal{R}^{m} f\right)^{q} d r\right)^{1 / q}\right) \\
& \leq C\left(\sup _{|z|<1 / 2}|f(z)|+\left(\int_{0}^{1}(1-r)^{q(m-\alpha)-1} M_{p}\left(r, \mathcal{R}^{m} f\right)^{q} d r\right)^{1 / q}\right)
\end{aligned}
$$

From this it follows that $\left\|\nabla_{T^{+}}^{k} f\right\|_{p, q, k / 2-\alpha}<\infty$.

The implication $(i) \Rightarrow($ iii $)$ holds also for $q=\infty$. Use Lemma 4.4 and (4.5). $(i i) \Rightarrow(i)$

A simple calculation shows that there are constant $d_{j}, j=0,1, \ldots, k$, such that

$$
\sum_{j=0}^{k} d_{j} \mathcal{R}^{k-j} f=\sum_{\delta \in C_{k}} \bar{T}_{\delta} T_{\delta} f
$$


Here, if $T_{\delta}=T_{1} \cdots T_{k}$, where $T_{m}, 1 \leq m \leq k$, are chosen among $T_{i, j}$ 's, then $\bar{T}_{\delta}=\bar{T}_{1} \cdots \bar{T}_{k}$. By using Lemma 4.2 we find that

$$
M_{p}^{p}\left(r, \bar{T}_{\delta} T_{\delta}\right) \leq \frac{C}{(1-r)^{1+k p / 2}} \int_{L_{r}}\left|T_{\delta} f(w)\right|^{p} d v(w),
$$

where

$$
L_{r}=\left\{z: \frac{1-r}{2}<1-|z|<2(1-r)\right\} .
$$

From (4.7) and (4.8) we conclude that

$$
\int_{0}^{1}(1-r)^{q(k-\alpha)-1} M_{p}\left(r, \sum_{j=0}^{k} d_{j} \mathcal{R}^{k-j}\right)^{q} d r \leq C \int_{0}^{1}(1-r)^{q(k / 2-\alpha)-1} M_{p}\left(r, \nabla_{T}^{k} f\right)^{q} d r .
$$

From this it follows that $f \in \Lambda_{\alpha}^{p, q}$. From (4.8) it follows that if $\left\|\nabla_{T}^{k} f\right\|_{p, \infty, k / 2-\alpha}<$ $\infty$, then $f \in \Lambda_{\alpha}^{p}$.

\section{Moduli OF SMOOThness AND MEAN GROWTH OF DERIVATIVES}

In this section we prove Theorem 1.5 and Theorem 1.6.

Proof of Theorem 1.5

Assume that $f$ is holomorphic in a neighborhood of the closed ball. For fixed $r \in(0,1)$ and $\zeta \in \mathbb{S}_{N}$, let $h(\theta)=f\left(r e^{i \theta} \zeta\right)$. By induction,

$$
\Delta_{t}^{n} h(\theta)=\int_{[0, t]^{n}} h^{(n)}\left(\theta+x_{1}+\ldots+x_{n}\right) d x_{1} \ldots x_{n} .
$$

Hence, by using (4.1) we get

$$
\begin{aligned}
i^{n} \mathcal{R}^{n} f\left(r e^{i \theta} \zeta\right) t^{n} & =h^{(n)}(\theta) t^{n} \\
& =\Delta_{t}^{n} h(\theta)-\int_{[0, t]^{n}}\left(h^{(n)}\left(\theta+x_{1}+\ldots+x_{n}\right)-h^{(n)}(\theta)\right) d x_{1} \ldots x_{n} .
\end{aligned}
$$

This implies that

$$
\begin{aligned}
\left|\mathcal{R}^{n} f\left(r e^{i \theta} \zeta\right)\right| t^{n} \leq & \left|\Delta_{t}^{n} f\left(r e^{i \theta} \zeta\right)\right| \\
& +\int_{[0, t]^{n}} \sup _{0<y<n t}\left|h^{(n+1)}(\theta+y)\right|\left(x_{1}+\ldots+x_{n}\right) d x_{1} \ldots d x_{n} \\
= & \left|\Delta_{t}^{n} f\left(r e^{i \theta} \zeta\right)\right|+\frac{n}{2} t^{n+1} \sup _{0<y<n t}\left|\mathcal{R}^{n+1} f\left(r e^{i(\theta+y)} \zeta\right)\right| .
\end{aligned}
$$

Hence, by Minkowski's inequality and the complex maximal theorem,

$$
t^{n} M_{p}\left(r, \mathcal{R}^{n} f\right) \leq\left\|\Delta_{t}^{n} f_{r}\right\|_{p}+C_{0} t^{n+1} M_{p}\left((3 r+1) / 4, \mathcal{R}^{n+1} f\right)
$$

provided $0<t \leq 1-r$. (See Lemma 3.1 of [16].) From this, the inequality

$$
M_{p}\left((3 r+1) / 4, \mathcal{R}^{n+1} f\right) \leq C_{1}(1-r)^{-1} M_{p}\left((1+r) / 2, \mathcal{R}^{n} f\right),
$$

and the inequality $\left\|\Delta_{t}^{n} f_{r}\right\|_{p} \leq\left\|\Delta_{t}^{n} f\right\|_{p}$, it follows that

$$
M_{p}\left(r, \mathcal{R}^{n} f\right) \leq t^{-n}\left\|\Delta_{t}^{n} f\right\|_{p}+C_{0} C_{1} t(1-r)^{-1} M_{p}\left((1+r) / 2, \mathcal{R}^{n} f\right) .
$$

Now let $q<\infty$ and

$$
A(r)=M_{p}^{q}\left(r, \mathcal{R}^{n} f\right) \psi(1-r) .
$$


Since $\psi(r / 2) \geq(1 / K) \psi(r)$, we have

$$
\begin{aligned}
A((1+r) / 2) & =M_{p}^{q}\left((1+r) / 2, \mathcal{R}^{n} f\right) \psi((1-r) / 2) \\
& \geq(1 / K) M_{p}^{q}\left((1+r) / 2, \mathcal{R}^{n} f\right) \psi(1-r)
\end{aligned}
$$

and therefore by using this and (5.1) we get

$$
A(r) \leq C_{2} t^{-n q}\left\|\Delta_{t}^{n} f\right\|_{p}^{q} \psi(1-r)+C_{3} K t^{q}(1-r)^{-q} A((1+r) / 2)
$$

for $0<t<1-r$. Now let $m>0$ be the smallest integer such that $2^{-m q} K C_{3} \leq 1 / 4$ and take $t=(1-r) / 2^{m}$. Then we have

$$
A(r) \leq C_{4}(1-r)^{-n q} \phi\left(2^{-m}(1-r)\right) \psi(1-r)+(1 / 4) A((1+r) / 2),
$$

where $\phi(t)=\left\|\Delta_{t}^{n} f\right\|_{p}^{q}$. Hence, by integration

$$
\int_{0}^{1} A(r) d r \leq C_{4} \int_{0}^{1} t^{-n q} \phi\left(2^{-m} t\right) \psi(t) d t+(1 / 2) \int_{1 / 2}^{1} A(r) d r
$$

and hence

$$
\begin{aligned}
(1 / 2) \int_{0}^{1} A(r) d r & \leq \int_{0}^{1} A(r) d r-(1 / 2) \int_{1 / 2}^{1} A(r) d r \\
& \leq C_{4} \int_{0}^{1} t^{-n q} \phi\left(2^{-m} t\right) \psi(t) d t \\
& =C_{5} \int_{0}^{2^{-m}} t^{-n q} \phi(t) \psi\left(2^{m} t\right) d t \\
& \leq C_{5} K^{m} \int_{0}^{1} t^{-n q} \phi(t) \psi(t) d t .
\end{aligned}
$$

This concludes the proof.

Corollary 5.1. If $\alpha>-1$, then

$$
\int_{r}^{1} M_{p}^{q}\left(\rho, \mathcal{R}^{n} f\right)(1-\rho)^{\alpha} d \rho \leq C \int_{0}^{1-r}\left[t^{-n}\left\|\Delta_{t}^{n} f\right\|_{p}\right]^{q} t^{\alpha} d t
$$

$(0<r<1)$, where $C$ is independent of $r$ and $f$.

Proof. For a fixed $r, 0<r<1$, we consider the function

$$
\psi(x)=x^{\alpha}, \quad \text { if } \quad 0<x<1-r, \quad \text { and } \quad \psi(x)=0, \quad \text { if } \quad 1-r<x<1 .
$$

Then $\psi$ satisfies (1.10) with $K=2^{\alpha}$, and $K$ is independent of $r$. Now (5.2) follows from (1.11).

Corollary 5.2. If $\alpha>-1$, then

$$
M_{p}\left(r, \mathcal{R}^{n} f\right) \leq C\left\{(1-r)^{-\alpha-1} \int_{0}^{1-r}\left[t^{-n}\left\|\Delta_{t}^{n} f\right\|_{p}\right]^{q} t^{\alpha} d t\right\}^{1 / q},
$$

where $C$ is independent of $r$ and $f$.

Proof. By the increasing property of $M_{p}\left(\rho, \mathcal{R}^{n}\right)$,

$$
M_{p}^{q}\left(r, \mathcal{R}^{n} f\right) \int_{r}^{1}(1-\rho)^{\alpha} d \rho \leq \int_{r}^{1} M_{p}^{q}\left(\rho, \mathcal{R}^{n}\right)(1-\rho)^{\alpha} d \rho,
$$

which, together with (5.2) gives (5.3). 
Having in mind Lemma 4.1, as a special case we have (1.12).

Theorem 1.6 is an easy consequence of Lemma 5.5 and Lemma 5.6 that we prove below.

Lemma 5.3. For $f \in H\left(\mathbb{B}_{N}\right)$ and $U \in \mathcal{L}\left(\mathbb{B}_{N}\right)$ we have

$$
\mathcal{R}^{n}(f \circ U)(w)=\left(\mathcal{R}^{n} f\right)(U w), \quad w \in \mathbb{B}_{N} .
$$

Proof. It is enough to consider the case $n=1$. We have $\mathcal{R} f(w)=f^{\prime}(w) w$, where $f^{\prime}(w)$ is the derivative of $f$ at $w$ treated as a linear operator on $\mathbb{C}^{N}$. Hence, by the chain rule,

$$
\mathcal{R}(f \circ U)(w)=(f \circ U)^{\prime}(w) w=f^{\prime}(U w) U w=(\mathcal{R} f)(U w) .
$$

Corollary 5.4. If $U \in \mathcal{L}\left(\mathbb{B}_{N}\right)$, then

$$
\mathcal{R}^{k} \Delta_{U}^{m} f=\Delta_{U}^{m} \mathcal{R}^{k} f, \quad k, m \geq 1 .
$$

Lemma 5.5. If $U \in \mathcal{L}\left(\mathbb{B}_{N}\right)$ and $\|U-I\|<1-r$, then for $f \in H\left(B_{N}\right)$ we have

$$
M_{p}\left(r, \Delta_{U}^{n} f\right) \leq C(1-r)^{n} M_{p}\left(r_{n}, \mathcal{R}^{n} f\right)
$$

where

$$
r_{n}=1-\frac{1-r}{2^{n}}
$$

Proof. (Induction on $n$.) Assume first that $f$ is holomorphic in a neighborhood of the closed ball. Let $\|U-I\|<\varepsilon$, where $\varepsilon$ is small enough. Then $|U r \zeta-r \zeta|<\varepsilon$ so, by the Lagrange theorem,

$$
|f(U r \zeta)-f(r \zeta)| \leq \sup _{|w-r \zeta|<\varepsilon,|w|<r}|\nabla f(w)| \varepsilon .
$$

Choose $\varepsilon=(1-r) / 2$. Since the inequalities $|w-r \zeta|<\varepsilon$ and $|w|<r$ imply

$$
\begin{aligned}
|w-\zeta| & \leq|w-r \zeta|+(1-r) \\
& \leq(3 / 2)(1-r) \\
& \leq(3 / 2)(1-|w|),
\end{aligned}
$$

we have that

$$
|f(U r \zeta)-f(r \zeta)| \leq \frac{1-r}{2} M_{*}(\nabla f)(\zeta),
$$

where $M_{*}$ is the non-tangential maximal function. Now the maximal theorem shows that

$$
M_{p}\left(r, \Delta_{U}^{1} f\right) \leq C(1-r)\|\nabla f\|_{p} .
$$

If $f \in H\left(\mathbb{B}_{N}\right)$ is arbitrary, then we apply this inequality to the function $f_{\rho}(z)=$ $f(\rho z), 0<\rho<1$, to get

$$
M_{p}\left(r \rho, \Delta_{U}^{1} f\right) \leq C(1-r) M_{p}(\rho, \nabla f) .
$$

Now take $\rho=(t+1) / 2$ and $r \rho=t$ for $0<t<1$. This implies, via Lemma 1.A.

$$
\begin{aligned}
M_{p}\left(t, \Delta_{U}^{1} f\right) & \leq C(1-t) M_{p}((t+1) / 2, \nabla f) \\
& \leq C(1-t) M_{p}((t+1) / 2, \mathcal{R} f) .
\end{aligned}
$$

This proves the lemma for $n=1$. 
Let $n \geq 2$. Then, by induction hypothesis, relation (5.4), and the case $n=1$,

$$
\begin{aligned}
M_{p}\left(r, \Delta_{U}^{n} f\right) & \leq C(1-r)^{n-1} M_{p}\left(r_{n-1}, \mathcal{R}^{n-1} \Delta_{U}^{1} f\right) \\
& =C(1-r)^{n-1} M_{p}\left(r_{n-1}, \Delta_{U}^{1} \mathcal{R}^{n-1} f\right) \\
& \leq C(1-r)^{n} M_{p}\left(\left(1+r_{n-1}\right) / 2, \mathcal{R}^{n} f\right) \\
& =C(1-r)^{n} M_{p}\left(r_{n}, \mathcal{R}^{n} f\right) .
\end{aligned}
$$

This completes the proof.

Lemma 5.6. If $f \in H^{p}\left(\mathbb{B}_{N}\right), U \in \mathcal{L}\left(\mathbb{B}_{N}\right)$, and $\|U-I\|<1-r, 1 / 4<r<1$, then

$$
\left\|\Delta_{U}^{n}\left(f-f_{r}\right)\right\|_{p} \leq C \int_{r}^{1}(1-s)^{n-1} M_{p}\left(s, \mathcal{R}^{n} f\right) d s .
$$

Proof. From the identity

$$
f(\zeta)-f(r \zeta)=\int_{r}^{1} \frac{1}{s} \mathcal{R} f(s \zeta) d s
$$

it follows that

$$
\left\|\Delta_{U}^{n}\left(f-f_{r}\right)\right\|_{p} \leq 4 \int_{r}^{1} M_{p}\left(s, \Delta_{U}^{n} \mathcal{R} f\right) d s .
$$

Hence, by Lemma 5.5 .

$$
\left\|\Delta_{U}^{n}\left(f-f_{r}\right)\right\|_{p} \leq C \int_{r}^{1}(1-s)^{n} M_{p}\left(s_{n}, \mathcal{R}^{n+1} f\right) d s,
$$

where

$$
s_{n}=1-\frac{1-s}{2^{n}} .
$$

Now we use the familiar estimate

$$
M_{p}(r, \mathcal{R} f) \leq M_{p}(r, \nabla f) \leq C(1-r)^{-1} M_{p}((1+r) / 2, f)
$$

to get

$$
M_{p}\left(s_{n}, \mathcal{R}^{n+1} f\right) \leq C(1-s)^{-1} M_{p}\left(s_{n+1}, \mathcal{R}^{n} f\right),
$$

which gives

$$
\left\|\Delta_{U}^{n}\left(f-f_{r}\right)\right\|_{p} \leq C \int_{r}^{1}(1-s)^{n-1} M_{p}\left(s_{n+1}, \mathcal{R}^{n} f\right) d s,
$$

Now the substitution $s_{n+1}=t$ completes the proof.

Proof of Theorem 1.6

In Corollary 2.5 it is shown that if

$$
\int_{0}^{1}(1-r)^{n-1} M_{p}\left(r, \mathcal{R}^{n} f\right) d r<\infty
$$

then $f \in H^{p}$. 
By using Lemma 5.5 and Lemma 5.6, (relations (5.5) and (5.6)), we get the inequality :

$$
\begin{aligned}
\omega_{n}^{+}(\delta, f)_{p} & =\sup _{\|U-I\|<\delta, U \in \mathcal{L}}\left\|\Delta_{U}^{n} f\right\|_{p} \\
& \leq \sup _{\|U-I\|<\delta, U \in \mathcal{L}}\left\|\Delta_{U}^{n} f_{1-\delta}\right\|_{p}+\sup _{\|U-I\|<\delta, U \in \mathcal{L}}\left\|\Delta_{U}^{n}\left(f-f_{1-\delta}\right)\right\|_{p} \\
& \leq C \int_{1-\delta}^{1}(1-r)^{n-1} M_{p}\left(r, \mathcal{R}^{n} f\right) d r
\end{aligned}
$$

\section{REMARKS}

For $f \in L^{1}(\sigma)$ and $z \in \mathbb{B}_{N}$ we define the Cauchy integral

$$
C f(z)=\int_{\mathbb{S}_{N}} f(\xi) \frac{d \sigma(\xi)}{(1-<z, \xi>)^{N}} .
$$

In 3 the authors obtained conditions on $f \in L^{1}(\sigma)$ sufficient to imply that $C f$ belongs to either the Besov space $\Lambda_{\alpha}^{p, p}$ or the Hardy-Sobolev space $H_{\alpha}^{p}$, where $1<p<\infty$ and $0<\alpha<\infty$. As a corollary of our results we have sufficient conditions different from those given in [3].

Theorem 6.1. Let $1<p<\infty, 0<\alpha<\infty$ and suppose that $n$ is an integer such that $0<\alpha<n$. Then a sufficient condition that $C f \in \Lambda_{\alpha}^{p, p}$ is that $f \in L^{p}(\sigma)$ and

$$
\int_{0}^{1} \frac{\left\|\Delta_{t}^{n} f\right\|_{p}^{p} d t}{t^{1+\alpha p}}<\infty
$$

A sufficient condition that $f \in H_{n}^{p}$ is that $f \in L^{p}(\sigma)$ and $\left\|\Delta_{t}^{n} f\right\|_{p}=\mathcal{O}\left(t^{n}\right)$.

\section{REFERENCES}

1. P.Ahern, R.Schneider, Holomorphic Lipschitz functions in pseudoconvex domains, Amer. J. Math., 101(1979), 543-565.

2. $\quad$ P.Ahern, J.Bruna, Maximal and area integral characterizations of Hardy-Sobolev spaces in the unit ball in $\mathbb{C}^{N}$, Revista Matematica Iberoamericana, 4(1988), 123-153.

3. P.Ahern, J.Bruna, Besov spaces, Sobolev spaces, and Cauchy integrals, Michigan Math. J., 39(1992),239-261.

4. O.Blasco, De.Souza, Spaces of analytic functions on the disc where the growth of $M_{p}(F, r)$ depends on a weight, J.Math.Anal.Appl., 147(1990), 580-598.

5. H.R.Cho, H.Koo, E.G.Kwon, Holomorphic mean Lipschitz spaces and Besov spaces on the unit ball in $\mathbb{C}^{N}$, (preprint).

6. H.R.Cho, K.Zhu, Holomorphic mean Lipschitz spaces and Hardy Sobolev spaces on the unit ball, (preprint).

7. P.L.Duren, Theory of $H^{p}$ spaces, Academic Press, New York, 1970; reprinted with supplement by Dover Publications, Mineola, N.Y., 2000.

8. G.H.Hardy, J.E.Littlewood, Some properties of fractional integrals , II, Math.Z., 34(1931), 403-439.

9. F.Holland, D.Walsh, Criteria for membership of the Besov space $B_{p, q}^{s}$, Math. Ann., 285(1989), 571-592.

10. M.Jevtić, M.Pavlović, On multipliers from $H^{p}$ to $l^{q}, 0<q<p<1$, Arch. Math., 56(1991), $174-180$

11. M.Jevtić, M.Pavlović, On the Hahn-Banach extension property in Hardy and mixed norm spaces on the unit ball, Monatsh. Math., 111(1991), 137-145.

12. M.Jevtić, M.Pavlović, Coefficient multipliers on spaces of analytic functions, Acta Sci. Math. (Szeged), 64(1998), 531-545.

13. E.G.Kwon, H.W.Koo, H.R.Cho, Holomorphic functions satisfying mean Lipschitz condition in the ball, J.Korean Math.Soc., 44(2007), 931-940. 
14. P.Oswald, On Besov-Hardy-Sobolev spaces of analytic functions in the unit disc, Czech. Math. J., 33(1983), 408-426.

15. M.Pavlović, Lipschitz spaces and spaces of harmonic functions in the unit disc, Michigan Math. J., 35(1988), 301-311.

16. M.Pavlović, On the moduli of continuity of $H^{p}$ functions with $0<p<1$, Proceedings of the Edinburgh Mathematical Society, 35(1992), 89-100.

17. M.Pavlović, Decomposition of $L^{p}$ and Hardy spaces of polyharmonic functions, J.Math.Anal.Appl., 216(1997), 499-509.

18. M.Pavlović, Introduction to function spaces on the disk, Matematički institut SANU, Beograd, 2004.

19. W.Rudin, Function Theory in the Unit Ball in $\mathbb{C}^{n}$, Springer-Verlag, New York, 1980.

20. K.Zhu, Spaces of Holomorphic Functions in the Unit Ball, Springer Verlag, New York, 2005.

21. A.Zygmund, Smooth functions, Duke Math. J., 12(1945), 47-76.

MatematičKi Fakultet, PP. 550, 11000 Belgrade, Serbia

E-mail address: jevtic@matf.bg.ac.yu

Faculty of Mathematics, Studentski trg 16, 11001 Belgrade, P.P. 550, Serbia

E-mail address: pavlovic@matf.bg.ac.rs 